A) Check for updates

Cite this: Nanoscale, 2020, 12, 11448

Received 14th March 2020,

Accepted 16th May 2020

DOI: 10.1039/d0nr02084b

rsc.li/nanoscale

\section{Transition from Schottky to Ohmic contacts in Janus MoSSe/germanene heterostructures $\uparrow$}

\author{
Ning Zhao and Udo Schwingenschlögl (D) *
}

\begin{abstract}
The performance of an electronic device based on a two-dimensional material is strongly affected by the contact with the metallic electrodes. In this article, we study the electronic properties of two-dimensional MoSSe in contact with a germanene electrode by first-principles calculations. The results show that the contact characteristics are significantly different for the two sides of MoSSe. Notably, for both sides in-plane tensile strain induces a transition from Schottky to Ohmic behavior. Increasing the thickness of MoSSe also leads to an Ohmic contact. We propose an effective route to high performance MoSSe electronic devices.
\end{abstract}

\section{Introduction}

Semiconductor/metal heterojunctions are of critical importance for electronic devices. In this context, van der Waals heterostructures consisting of stacked two-dimensional materials have opened up a new paradigm to design the interface properties. ${ }^{1-5}$ As an Ohmic contact with low resistance is the ultimate goal to achieve high performance devices, ${ }^{6}$ the Schottky barrier height (SBH; energy barrier that must be overcome by charge carriers to cross the interface; positive value indicating formation of a Schottky instead of an Ohmic contact) becomes a key parameter., ${ }^{7,8}$

Two-dimensional Janus MoSSe has first been prepared by Lu and coworkers in 2017. ${ }^{9}$ Similar to $\mathrm{MoS}_{2}$, its band gap and carrier mobility show a distinct thickness-dependence. ${ }^{10}$ On the other hand, the intrinsic dipole of MoSSe (structural symmetry of $\mathrm{MoS}_{2}$ is broken) modifies the electronic properties. ${ }^{11}$ A number of theoretical studies have shown that two-dimensional Janus materials perform well in gas sensors, ${ }^{12,13}$ photocatalytic water splitting, ${ }^{14-16}$ field-effect transistors, ${ }^{17}$ and solar cells. ${ }^{18,19}$ However, practical use of MoSSe in a device will

Physical Science and Engineering Division (PSE), King Abdullah University of Science and Technology (KAUST), Thuwal, 23955-6900, Saudi Arabia.

E-mail: udo.schwingenschlogl@kaust.edu.sa

$\dagger$ Electronic supplementary information (ESI) available. See DOI: 10.1039/ d0nr02084b require a contact with metal electrodes to enable injection of carriers. ${ }^{20}$ For this reason, we investigate in the present work van der Waals heterostructures constructed from MoSSe (considering both the $\mathrm{S}$ and Se sides) and germanene (Ge). Ge is chosen as electrode, because it is a Dirac metal with perfect lattice match to MoSSe. ${ }^{21}$ Employing first-principles calculations, we explore the electronic properties of the heterostructures, focusing on the SBH and band alignment, which are critical for the device performance. We analyze how Ge interacts with the different sides of MoSSe and determine the resulting contact characteristics.

\section{Computational methods}

All the calculations are performed using the Vienna $A b$ initio Simulation Package. ${ }^{22}$ The generalized gradient approximation (GGA) of Perdew-Burke-Ernzerhof ${ }^{23}$ is applied to the exchange correlation functional and the DFT-D3 correction $^{24}$ is used to account for the van der Waals interaction. The plane-wave cutoff energy is set to $500 \mathrm{eV}$ and a Monkhorst-Pack ${ }^{25} 9 \times 9 \times 1$ k-mesh is utilized for Brillouin-zone integration $(5 \times 5 \times 1$ in the structural relaxation). The convergence criteria are set to $0.01 \mathrm{eV} \AA^{-1}$ for the maximal residual force and $10^{-6} \mathrm{eV}$ for the total energy. Combining a $4 \times 4 \times 1$ supercell of Ge with 32 germanium atoms and a $5 \times 5 \times 1$ supercell of MoSSe with 75 atoms leads to a small lattice mismatch of less than $0.5 \%$. In each heterostructure a vacuum layer of $15 \AA$ thickness is added for ensuring that there is no interaction between periodic images in the out-of-plane direction.

\section{Results and discussion}

We first investigate the geometries of $\mathrm{Ge}$ and monolayer MoSSe, obtaining the lattice parameters 4.04 and $3.25 \AA$ after optimization, respectively, in good agreement with previous studies. ${ }^{26,27}$ In contrast to $\mathrm{MoS}_{2}$, MoSSe has an intrinsic dipole pointing from Se to $\mathrm{S}$ with a calculated dipole moment of $D=$ 
0.25 Debye, which is also consistent with previous results. ${ }^{28}$ Both the $\mathrm{S}$ and Se sides of MoSSe can be in contact with Ge to form a heterostructure, namely, SeMoS/Ge with S-Ge interface and SMoSe/Ge with Se-Ge interface. To verify stability of the heterostructures, the binding energy

$$
E_{\mathrm{b}}=\left[E_{\mathrm{Ge}}+E_{\mathrm{MoSSe}}-E_{\text {hetero }}\right] / N
$$

is calculated, where $E_{\mathrm{Ge}}, E_{\mathrm{Mosse}}$, and $E_{\text {hetero }}$ are the total energies of Ge (consisting of $N$ atoms), MoSSe, and the heterostructure. By configuration scanning (see Fig. S1† for the considered stacking configurations), the energetically favorable heterostructures are determined. They are shown in Fig. 1(a and b). We obtain equilibrium interlayer distances of $2.92 \AA$ for SeMoS/Ge and $3.07 \AA$ for SMoSe/Ge, see Fig. 1(c and d). (a) SeMoS/Ge
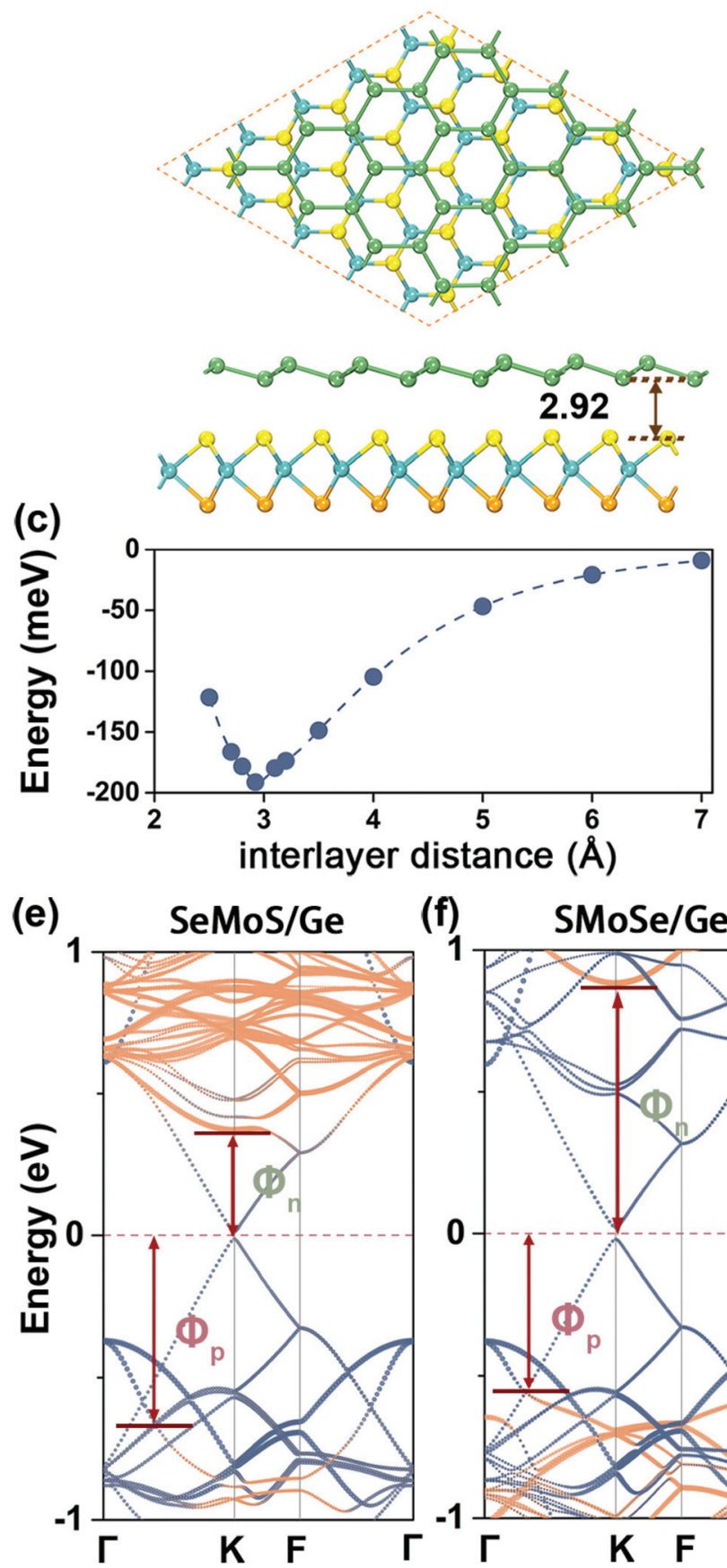

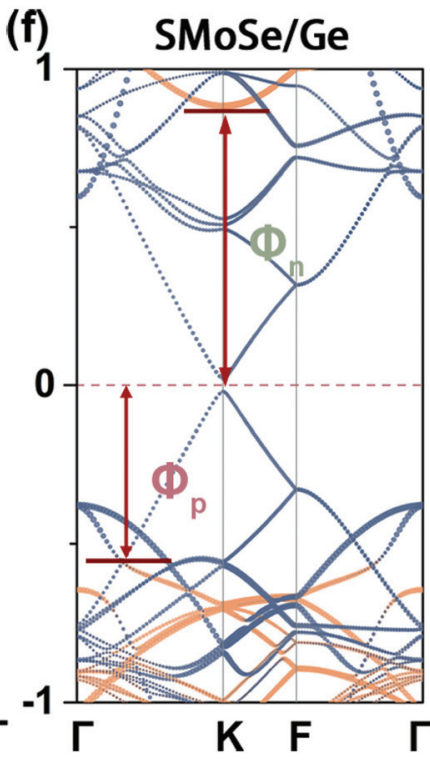

(b) SMoSe/Ge

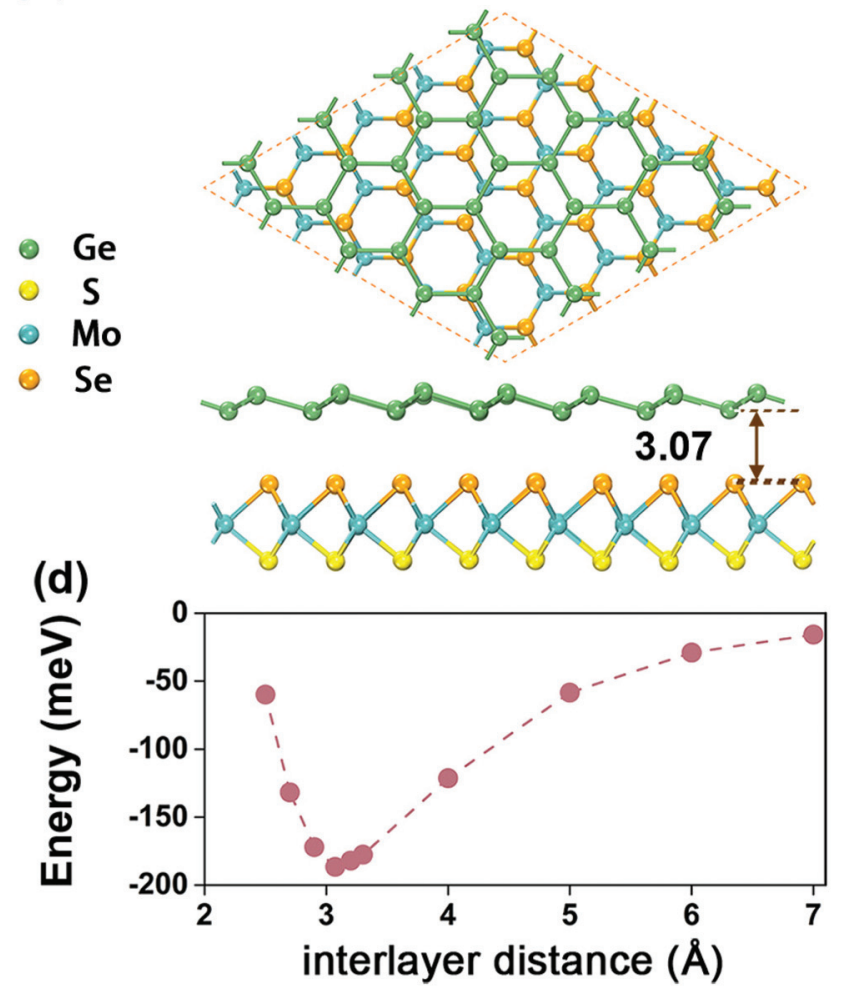

(g)

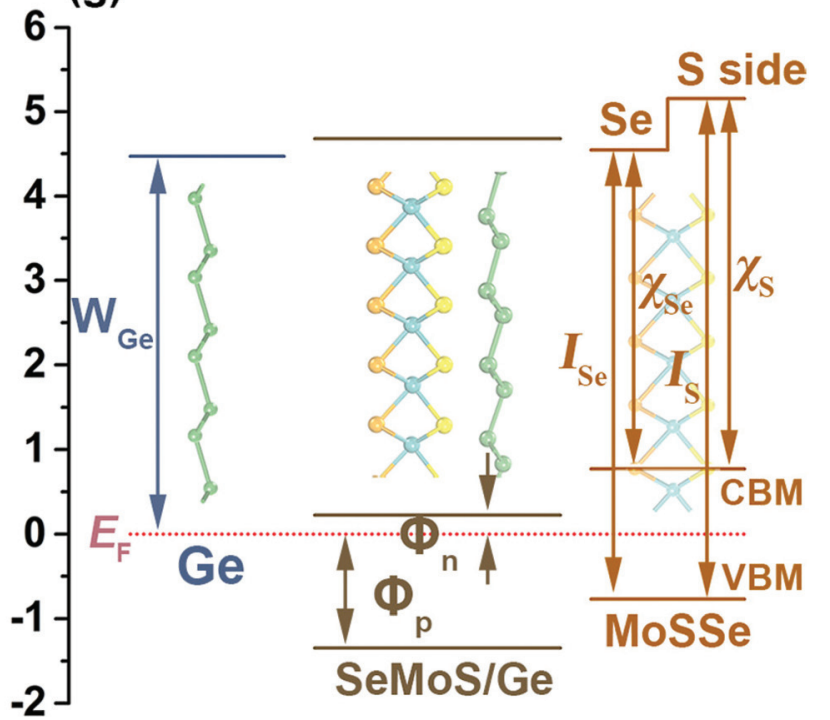

Fig. 1 Top and side views of (a) SeMoS/Ge and (b) SMoSe/Ge. The dependence of the binding energy on the interlayer distance is shown in (c) and (d). Weighted band structures are given in (e) and (f) with orange and blue colors indicating the contributions of Ge and MoSSe, respectively. Corresponding densities of states are shown in Fig. S5. $\dagger$ (g) Energy band diagram of Ge, monolayer MoSSe, and SeMoS/Ge. 
The corresponding weighted band structures are shown in Fig. 1(e and f). Orange and blue colors represent the contributions of MoSSe and Ge, respectively. Comparing to the pristine component materials (Fig. S2 $\dagger$ ), the band structures of the heterostructures are more than simple superpositions due to hybridization of the atomic orbitals at the interfaces. The effects of spin-orbital coupling have been tested carefully for MoSSe and Ge, showing no significant influence on the band dispersions (Fig. S3†). Therefore, spin-orbit coupling is not taken into account for the heterostructures. According to Fig. 1 (e and f), a band gap of 25 and $41 \mathrm{meV}$ opens at the Dirac point for SeMoS/Ge and SMoSe/Ge, respectively. Interestingly, the band gap of the semiconducting layer (measured from the highest valence to the lowest conduction state dominated by MoSSe) in SeMoS/Ge is $1.00 \mathrm{eV}$, i.e., smaller than in the case of pristine MoSSe (1.55 eV), while in SMoSe/Ge it is $1.45 \mathrm{eV}$. This trend does not reflect the typical behavior of two-dimensional heterostructures ${ }^{29}$ due to the fact that the strong hybridization seen in Fig. 1(e and $\mathrm{f}$ ) between the component layers is unusal. $^{30-32}$

Our results show that an n-type Schottky contact is formed in SeMoS/Ge and a p-type Schottky contact in SMoSe/Ge. The $\mathrm{SBHs}$ of the heterostructures are given by $^{33}$

$$
\begin{aligned}
& \Phi_{\mathrm{n}}=W_{\mathrm{SeMoS} / \mathrm{Ge}}-\chi_{\mathrm{S}}, \Phi_{\mathrm{p}}=I_{\mathrm{S}}-W_{\mathrm{SeMoS} / \mathrm{Ge}} \\
& \Phi_{\mathrm{n}}=W_{\mathrm{SMoSe} / \mathrm{Ge}}-\chi_{\mathrm{Se}}, \Phi_{\mathrm{p}}=I_{\mathrm{Se}}-W_{\mathrm{SMoSe} / \mathrm{Ge}}
\end{aligned}
$$

where $W_{\text {SeMoS/Ge }}$ and $W_{\text {SMoSe/Ge }}$ are the work functions. Moreover, $\chi_{\mathrm{S} / \mathrm{Se}}$ and $I_{\mathrm{S} / \mathrm{Se}}$ denote the electron affinity and ionization potential of the S/Se side of MoSSe, respectively. In Fig. 1(g) we show for SeMoS/Ge (S side in contact with Ge), the energy band diagram at large interlayer distance (no interaction
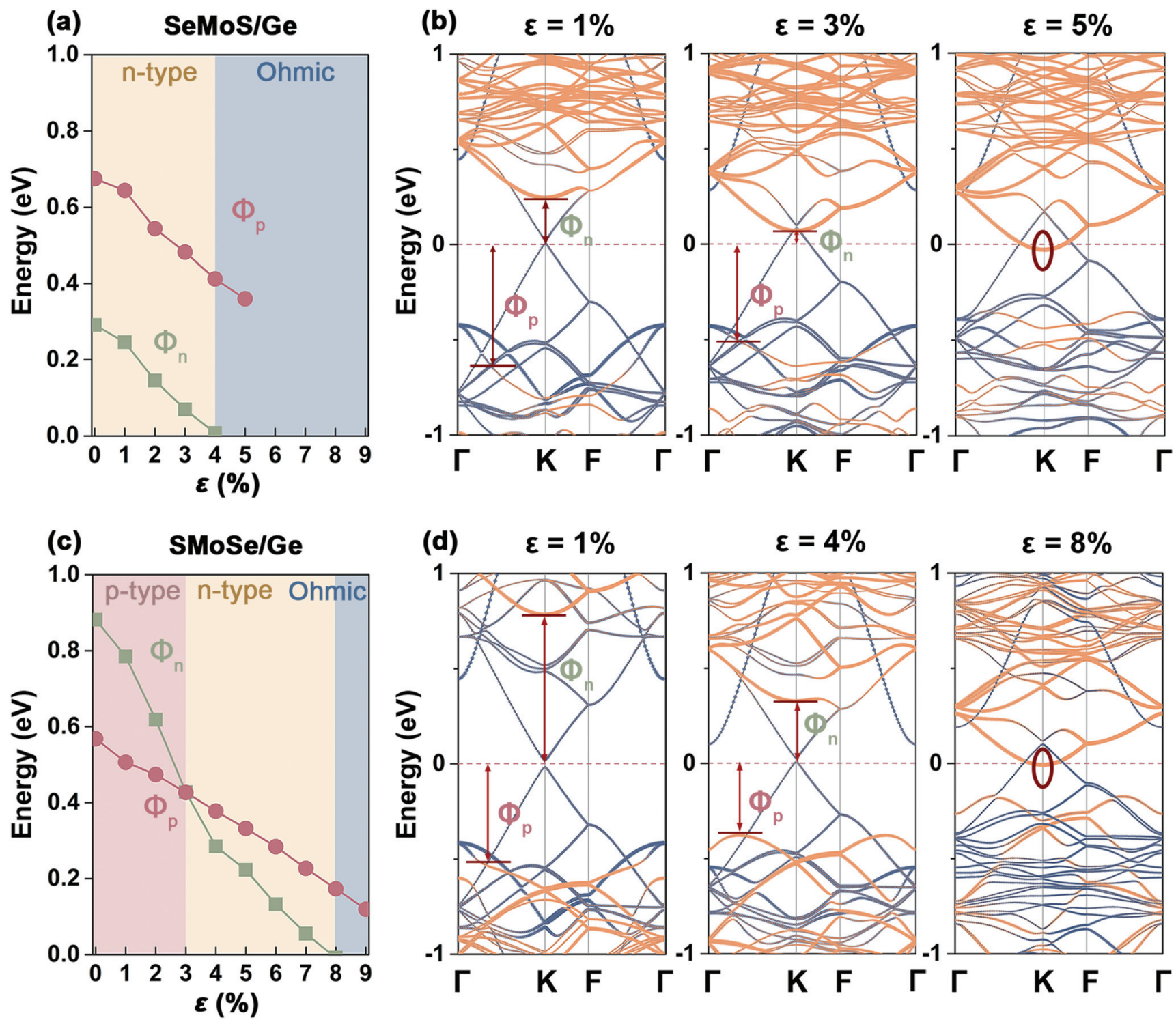

Fig. $2 \mathrm{SBHs}$ of (a) SeMoS/Ge and (c) SMoSe/Ge as functions of strain. Weighted band structures are given in (b) and (d) for different strains with orange and blue colors indicating the contributions of Ge and MoSSe, respectively. Corresponding densities of states are shown in Fig. S5. $\dagger$ 
between the components), i.e., there is no effect of charge transfer. A quantitative calculation at an interlayer distance of $7 \AA$ results in $W_{\text {SeMos } / \mathrm{Ge}}=4.68 \mathrm{eV}, I_{\mathrm{S}}=5.93 \mathrm{eV}$, and $\chi_{\mathrm{S}}=4.38$ $\mathrm{eV}$. Therefore, $\Phi_{\mathrm{n}}=0.30 \mathrm{eV}$ and $\Phi_{\mathrm{p}}=1.25 \mathrm{eV}$, consistent with the values measured in the band structures (Fig. S4; $\uparrow 0.23$ and $1.34 \mathrm{eV}$ ).

A tunable $\mathrm{SBH}$ is essential to improve the transition of charge carriers between the two components of a heterostructure. To this aim, we explore the effect of tensile strain $(\varepsilon)$, finding that for increasing $\varepsilon$ the interaction between MoSSe and Ge intensifies. According to Fig. 2(a), both $\Phi_{\mathrm{n}}$ and $\Phi_{\mathrm{p}}$ of SeMoS/Ge decrease. The n-type $\mathrm{SBH}$ vanishes at $\varepsilon \sim 4 \%$, i.e., the contact becomes Ohmic. Band structures at different strains are shown in Fig. 2(b), demonstrating that the conduction band minimum of MoSSe shifts dramatically to lower energy (relative to the Fermi energy) for increasing $\varepsilon$, while the Dirac point of Ge shifts to higher energy similar to isolated Ge. ${ }^{34}$ A more complicated situation is encountered in SMoSe/ Ge, see Fig. 2(c). Faster decrease of $\Phi_{\mathrm{n}}$ than $\Phi_{\mathrm{p}}$ results in an n-type $\mathrm{SBH}$ for strain above $\varepsilon \sim 3 \%$ and an Ohmic contact is achieved at $\varepsilon \sim 8 \%$. According to the band structures in Fig. 2(d), similar to SeMoS/Ge, for increasing $\varepsilon$ the Dirac point of Ge shifts to higher energy. We have confirmed our conclusions for the non-local van der Waals method (otherwise same computational setup), ${ }^{35-38}$ finding that the interlayer distance increases significantly from $2.92 \AA$ to $3.50 \AA$ for SeMoS/ Ge and from $3.07 \AA$ to $3.67 \AA$ for SMoSe/Ge, while $\Phi_{\mathrm{n}}(0.36 \mathrm{eV}$ instead of $0.29 \mathrm{eV}$ for SeMoS/Ge; $0.89 \mathrm{eV}$ instead of $0.88 \mathrm{eV}$ for SMoSe/Ge) and $\Phi_{\mathrm{p}}(1.02 \mathrm{eV}$ instead of $0.67 \mathrm{eV}$ for SeMoS/Ge; $0.71 \mathrm{eV}$ instead of $0.57 \mathrm{eV}$ for SMoSe/Ge) increase only slightly and the contact type is not modified.

To obtain insight into the Schottky-to-Ohmic transition, the strain-dependence of the electrostatic potential is addressed in Fig. 3(a and e) (vacuum level set to zero energy). While the shortened interlayer distance of the heterostructures under tensile strain results in minor shifts of the peaks, the heights of the peaks decrease gradually. We observe in Fig. 3(b and $\mathrm{f}$ ) that the charge transfer from Ge to MoSSe (positive $\Delta q$ ) gener- (a)
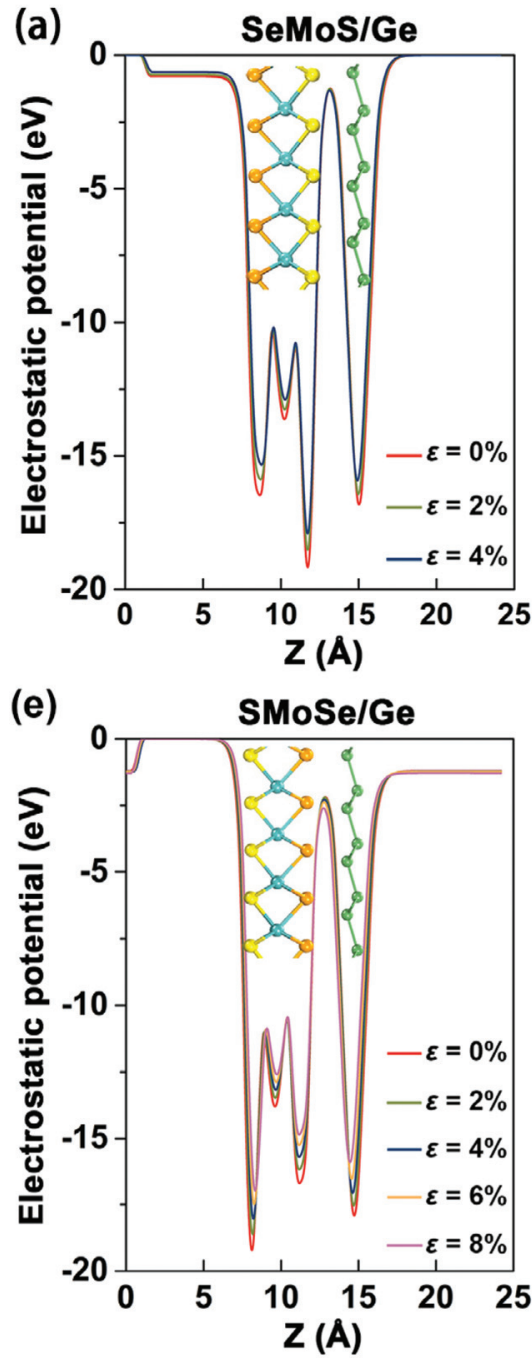

(b)

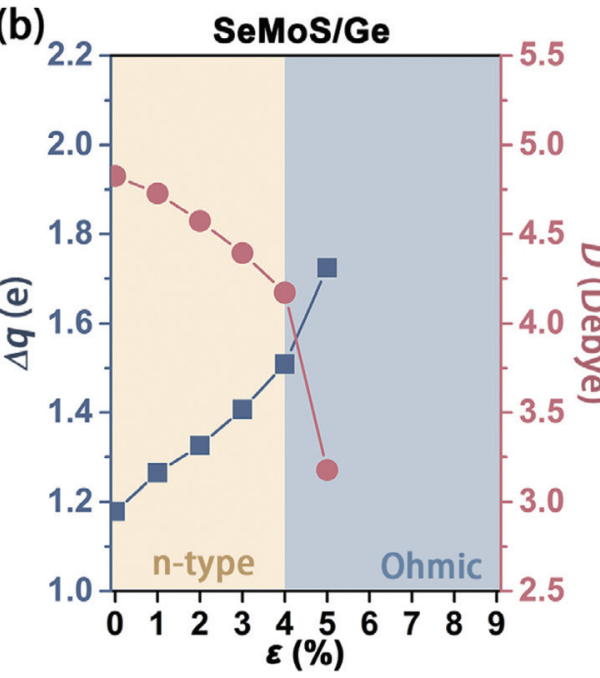

(f)

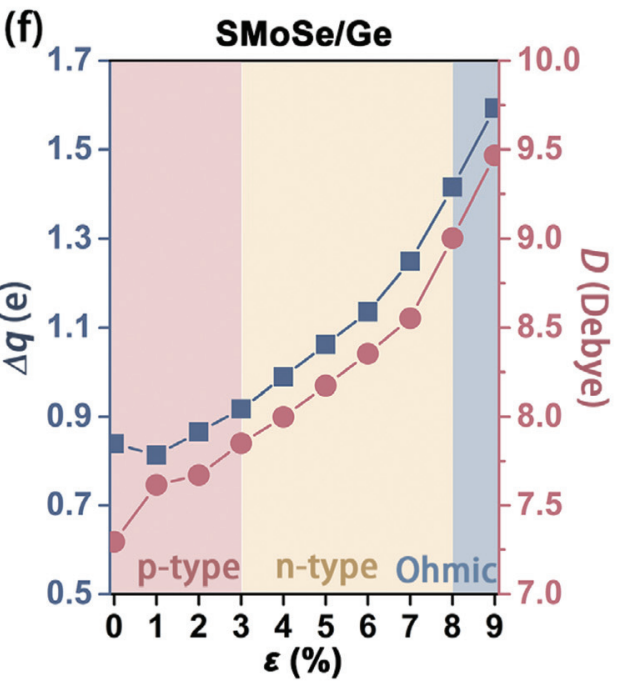

(c)

(d)
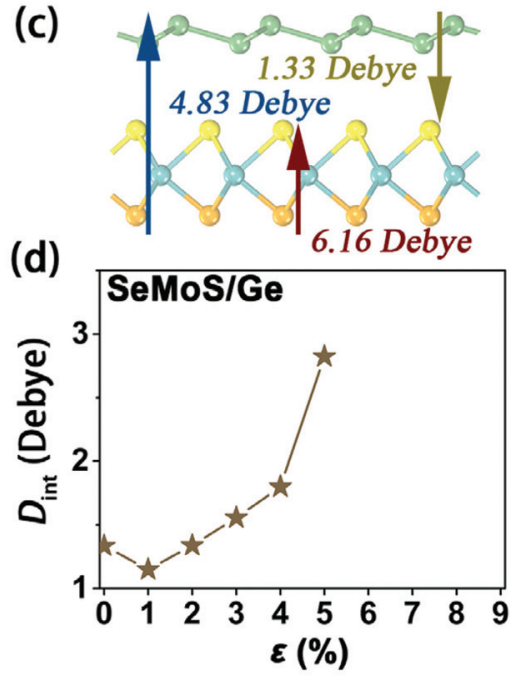

(g)
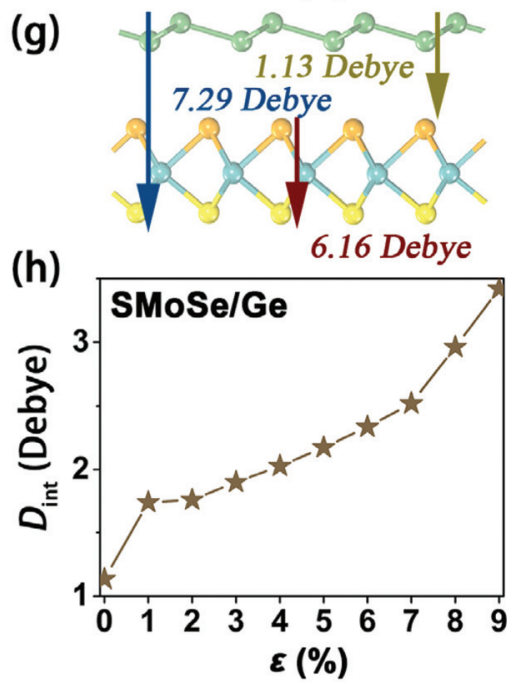

Fig. 3 Electrostatic potentials of (a) SeMoS/Ge and (e) SMoSe/Ge for different strains. Corresponding charge transfers and dipole moments as functions of strain are given in (b) and (f), dipole moments without strain in (c) and (g), and interface dipole moments as functions of strain in (d) and (h). 


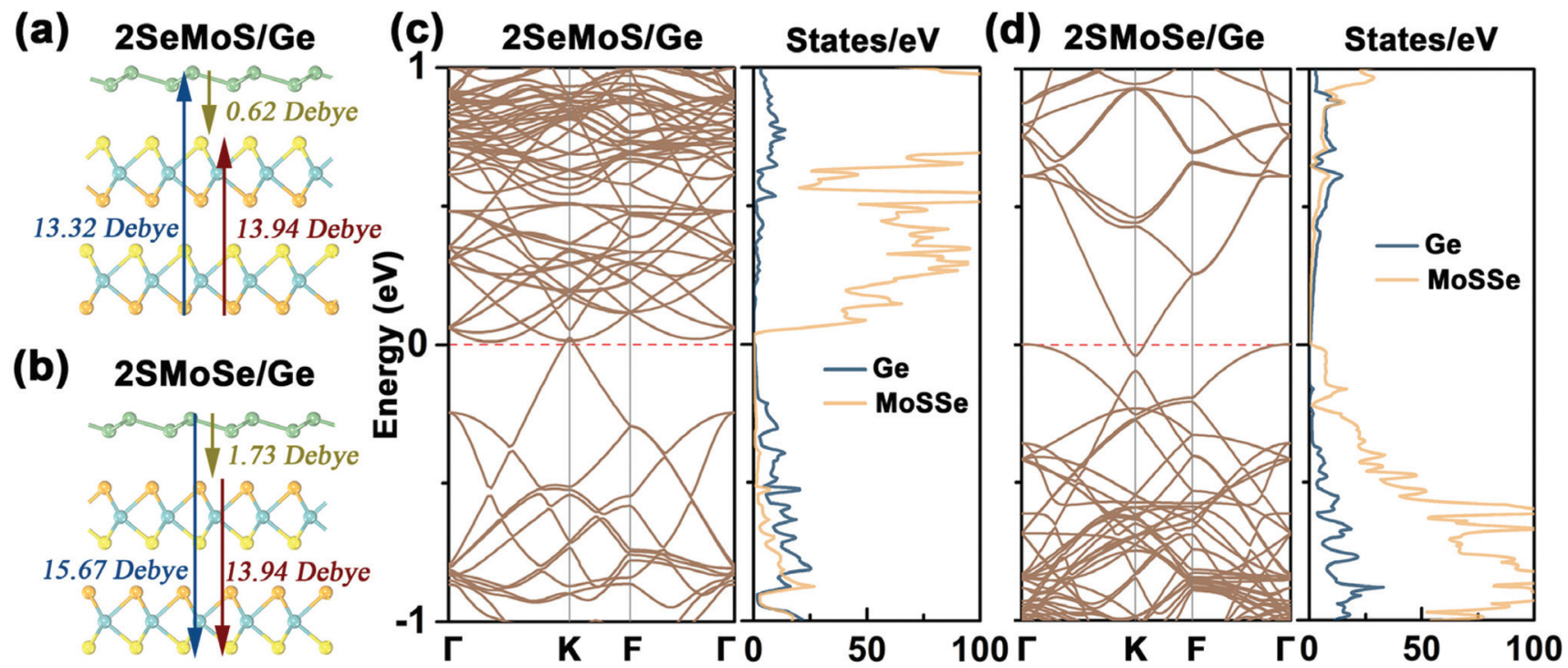

Fig. 4 Dipole moments of (a) 2SeMoS/Ge and (b) 2SMoSe/Ge. Corresponding band structures and densities of states are shown in (c) and (d).

ally increases under tensile strain (faster for higher strain). The value is larger for $\mathrm{SeMoS} / \mathrm{Ge}$ than $\mathrm{SMoSe} / \mathrm{Ge}$ due to the fact that $\mathrm{S}$ is more electronegative than Se. In addition, Fig. 3(b and $\mathrm{f}$ ) shows that the total dipole moment points from Se to $\mathrm{S}$ $(D>0)$ for both heterostructures, being larger for SMoSe/Ge than SeMoS/Ge. We have $D=D_{0}+D_{\text {int }}$, where $D_{0}$ is the intrinsic dipole moment of MoSSe (6.16 Debye for the $5 \times 5 \times 1$ supercell) and $D_{\text {int }}$ is the interlayer dipole moment (1.33 and 1.13 Debye for SeMoS/Ge and SMoSe/Ge without strain, see Fig. 3(c and $\mathrm{g}$ )). $D$ of $\mathrm{SeMoS} / \mathrm{Ge}$ decreases under tensile strain, see Fig. 3(b), whereas $D$ of SMoSe/Ge increases, see Fig. 3(f). Though the direction of $D$ is different in the two heterostructures (pointing from MoSSe to Ge for SeMoS/Ge and from Ge to MoSSe for SMoSe/Ge), $D_{\text {int }}$ always points from Ge to MoSSe. $D_{\text {int }}$ increases under tensile strain, as $\Delta q$ increases, see Fig. $3\left(\mathrm{~d}\right.$ and h). At $D_{\text {int }}=2.82$ Debye for $\mathrm{SeMoS} / \mathrm{Ge}$ and $D_{\text {int }}=$ 2.96 Debye for SMoSe/Ge an Ohmic contact is achieved. Therefore, $D_{\text {int }}$ controls the SBH.

As not only monolayer MoSSe but also bilayer MoSSe has an intrinsic dipole, we next address heterostructures of bilayer MoSSe with Ge, see Fig. 4(a and b). As expected, $D_{0}$ increases in the case of bilayer MoSSe (13.94 Debye for the $5 \times 5 \times 1$ supercell) as compared to the case of monolayer MoSSe. While $D_{\text {int }}$ still points from Ge to MoSSe, it decreases to 0.62 Debye for $2 \mathrm{SeMoS} / \mathrm{Ge}$ and increases to 1.73 Debye for $2 \mathrm{SMoSe} / \mathrm{Ge}$. The charge transfer is still directed from Ge to MoSSe with values of 1.15 e for $2 \mathrm{SeMoS} / \mathrm{Ge}$ and 0.78 e for $2 \mathrm{SMoSe} / \mathrm{Ge}$, which are similar to the case of monolayer MoSSe. According to Fig. 4(c and d), both heterostructures have a gapped Dirac cone near the Fermi energy, shifted as expected from the dipole (build-in electric field ${ }^{39}$ ). We obtain for $2 \mathrm{SeMoS} / \mathrm{Ge} \Phi_{\mathrm{n}}=$ $0.01 \mathrm{eV}$, i.e., an almost Ohmic contact, while for $2 \mathrm{SMoSe} / \mathrm{Ge}$ the bands of the semiconducting layer touch the Fermi energy, i.e., an Ohmic contact is formed.

\section{Conclusion}

We have systematically studied heterostructures of two-dimensional Janus MoSSe with germanene (Ge). An n-type Schottky contact is found to be formed for SeMoS/Ge and a p-type Schottky contact for SMoSe/Ge. A transition from Schottky to Ohmic behavior occurs under tensile strain ( $4 \%$ for SeMoS/Ge, $\sim 8 \%$ for $\mathrm{SMoSe} / \mathrm{Ge}$ ), which is explained by modifications of the interface dipole. Of great interest from a technological perspective is the observation that an increased thickness of MoSSe (bilayer sufficient) directly results in an Ohmic contact. Our investigation thus demonstrates that $\mathrm{Ge}$ is an ideal electrode for contacting two-dimensional Janus MoSSe in electronic devices.

\section{Conflicts of interest}

There are no conflicts to declare.

\section{Acknowledgements}

The research reported in this publication was supported by funding from King Abdullah University of Science and Technology (KAUST).

\section{References}

1 A. K. Geim and I. V. Grigorieva, van der Waals Heterostructures, Nature, 2013, 449, 419-425.

2 X. Hong, J. Kim, S.-F. Shi, Y. Zhang, C. Jin, Y. Sun, S. Tongay, J. Wu, Y. Zhang and F. Wang, Ultrafast Charge Transfer in Atomically Thin $\mathrm{MoS}_{2} / \mathrm{WS}_{2}$ Heterostructures, Nat. Nanotechnol., 2014, 9, 682-686. 
3 X. Cui, G.-H. Lee, Y. D. Kim, G. Arefe, P. Y. Huang, C.-H. Lee, D. A. Chenet, X. Zhang, L. Wang, F. Ye, F. Pizzocchero, B. S. Jessen, K. Watanabe, T. Taniguchi, D. A. Muller, T. Low, P. Kim and J. Hone, Multi-Terminal Transport Measurements of $\mathrm{MoS}_{2}$ Using a van der Waals Heterostructure Device Platform, Nat. Nanotechnol., 2015, 10, 534-540.

4 N. S. Novoselov, A. Mishchenko, A. Carvalho and A. H. Castro Neto, 2D Materials and van der Waals Heterostructures, Science, 2016, 353, aac9439.

5 S. Gao, L. Yang and C. D. Spataru, Interlayer Coupling and Gate-Tunable Excitons in Transition Metal Dichalcogenide Heterostructures, Nano Lett., 2017, 17, 7809-7813.

6 F. A. Kröger, G. Diemer and H. A. Klasens, Nature of an Ohmic Metal-Semiconductor Contact, Phys. Rev., 1956, 103, 279.

7 R. T. Tung, The Physics and Chemistry of the Schottky Barrier Height, Appl. Phys. Rev., 2014, 1, 011304.

8 Y. Li, J. Wang, B. Xhou, F. Wang, Y. Miao, J. Wei, B. Zhang and K. Zhang, Tunable Interlayer Coupling and Schottky Barrier in Graphene and Janus MoSSe Heterostructures by Applying an External Field, Phys. Chem. Chem. Phys., 2018, 20, 24109-24116.

9 A.-Y. Lu, H. Zhu, J. Xiao, C.-P. Chuu, Y. Han, M.-H. Chiu, C.-C. Cheng, C.-W. Yang, K.-H. Wei, Y. Yang, Y. Wang, D. Sokaras, D. Nordlund, P. Yang, D. A. Muller, M.-Y. Chou, X. Zhang and L.-J. Li, Janus Monolayers of Transition Metal Dichalcogenides, Nat. Nanotechnol., 2017, 12, 744-749.

10 W.-J. Yin, B. Wen, G.-Z. Nie, X.-L. Wei and L.-M. Liu, Tunable Dipole and Carrier Mobility for a Few Layer Janus MoSSe Structure, J. Mater. Chem. C, 2018, 6, 1693-1700.

11 C. Xia, W. Xiong, J. Du, T. Wang, Y. Peng and J. Li, Universality of Electronic Characteristics and Photocatalyst Applications in the Two-Dimensional Janus Transition Metal Dichalcogenides, Phys. Rev. B, 2018, 98, 165424.

12 C. Jin, X. Tang, X. Tan, S. C. Smith, Y. Dai and L. Kou, A Janus MoSSe Monolayer: A Superior and Strain-Sensitive Gas Sensing Material, J. Mater. Chem. A, 2019, 7, 1099-1106.

13 R. Chaurasiya and A. Dixit, Defect Engineered MoSSe Janus Monolayer as a Promising Two-Dimensional Material for $\mathrm{NO}_{2}$ and NO Gas Sensing, Appl. Surf. Sci., 2019, 490, 204-219.

14 Z. Guan, S. Ni and S. Hu, Tunable Electronic and Optical Properties of Monolayer and Multilayer Janus MoSSe and a Photocatalyst for Solar Water Splitting: A First-Principles Study, J. Phys. Chem. C, 2018, 122, 6209-6216.

15 X. Ma, X. Wu, H. Wang and Y. Wang, A Janus MoSSe Monolayer: A Potential Wide Solar Spectrum WaterSplitting Photocatalyst with a Low Carrier Recombination Rate, J. Mater. Chem. A, 2018, 6, 2295-2301.

16 W. Yin, B. Wen, Q. Ge, D. Zou, Y. Xu, M. Liu, X. Wei, M. Chen and X. Fan, Role of Intrinsic Dipole on Photocatalytic Water Splitting for Janus MoSSe/Nitrides Heterostructure: A First-Principles Study, Prog. Nat. Sci., 2019, 29, 335-340.

17 X. Tang, S. Li, Y. Ma, A. Du, T. Liao, Y. Gu and L. Kou, Distorted Janus Transition Metal Dichalcogenides: Stable
Two-Dimensional Materials with Sizable Band Gap and Ultrahigh Carrier Mobility, J. Phys. Chem. C, 2018, 122, 19153-19160.

18 R. Chaurasiya, G. K. Gupta and A. Dixit, Ultrathin Janus WSSe Buffer Layer for W(S/Se $)_{2}$ Absorber Based Solar Cells: A hybrid, DFT and Macroscopic, Simulation Studies, Sol. Energy Mater. Sol. Cells, 2019, 201, 110076.

19 M. Idrees, H. U. Din, R. Ali, G. Rehman, T. Hussain, C. V. Nguyen, L. Ahmad and B. Amin, Optoelectronic and Solar Cell Applications of Janus Monolayers and Their van der Waals Heterostructures, Phys. Chem. Chem. Phys., 2019, 21, 18612-18621.

20 A. Allain, J. Kang, K. Banerjee and A. Kis, Electrical Contacts to Two-Dimensional Semiconductors, Nat. Mater., 2015, 14, 1195-1205.

21 J.-A. Yan, S.-P. Gao, R. Stein and G. Coard, Tuning the Electronic Structures of Silicene and Germanene by Biaxial Strain and Electric Field, Phys. Rev. B: Condens. Matter Mater. Phys., 2015, 91, 245403.

22 G. Kresse and D. Joubert, From Ultrasoft Pseudopotentials to the Projector Augmented-Wave Method, Phys. Rev. B: Condens. Matter Mater. Phys., 1999, 59, 1758-1775.

23 J. P. Perdew, K. Burke and M. Ernzerhof, Generalized Gradient Approximation Made Simple, Phys. Rev. Lett., 1996, 77, 3865-3868.

24 S. Grimme, J. Antony, S. Ehrlich and H. Krieg, A Consistent and Accurate $\mathrm{Ab}$ Initio Parametrization of Density Functional Dispersion Correction (DFT-D) for the 94 Elements H-Pu, J. Chem. Phys., 2010, 132, 154104.

25 H. J. Monkhorst and J. D. Pack, Special Points for BrillouinZone Integrations, Phys. Rev. B: Solid State, 1976, 13, 5188-5192.

26 L. Matthes, O. Pulci and F. Bechstedt, Massive Dirac Quasiparticles in the Optical Absorbance of Graphene, Silicene, Germanene, and Tinene, J. Phys.: Condens. Matter, 2013, 25, 395305.

27 S. Tao, B. Xu, J. Shi, S. Zhong, X. Lie, G. Liu and M. Wu, Tunable Dipole Moment in Janus Single-Layer MoSSe Via Transition-Metal Atom Adsorption, J. Phys. Chem. C, 2019, 123, 9059-9065.

28 F. Li, W. Wei, P. Zhao, B. Huang and Y. Dai, Electronic and Optical Properties of Pristine and Vertical and Lateral Heterostructures of Janus MoSSe and WSSe, J. Phys. Chem. Lett., 2017, 8, 5959-5965.

29 T. Shen, J.-C. Ren, X. Liu, S. Li and W. Liu, van der Waals Stacking Induced Transition from Schottky to Ohmic Contacts: 2D Metals on Multilayer InSe, J. Am. Chem. Soc., 2019, 141, 3110-3115.

30 W. Xiong, C. Xia, X. Zhao, T. Wang and Y. Jia, Effects of Strain and Electric Field on Electronic Structures and Schottky Barrier in Graphene and SnS Hybrid Heterostructures, Carbon, 2016, 109, 737-746.

31 S. Sattar and U. Schwingenschlögl, Electronic Properties of Graphene-PtSe ${ }_{2}$ Contacts, ACS Appl. Mater. Interfaces, 2017, 9, 15809-15813.

32 T. Jing, D. Liang, J. Hao, M. Deng and S. Cai, Interface Schottky Barrier in $\mathrm{Hf}_{2} \mathrm{NT}_{2} / \operatorname{MoSSe}(\mathrm{T}=\mathrm{F}, \mathrm{O}, \mathrm{OH} ; \mathrm{M}=\mathrm{Mo}$, 
W) Heterostructures, Phys. Chem. Chem. Phys., 2019, 21, 5394-5401.

33 J. Bardeen, Surface States and Rectification at a Metal Semi-Conductor Contact, Phys. Rev., 1974, 71, 717-727.

34 Y. Wang and Y. Ding, Strain-Induced Self-Doping in Silicene and Germanene from First-Principles, Solid State Commun., 2013, 155, 6-11.

35 M. Dion, H. Rydberg, E. Schröder, D. C. Langreth and B. I. Lundqvist, van der Waals Density Functional for General Geometries, Phys. Rev. Lett., 2004, 92, 246401.

36 G. Román-Pérez and J. M. Soler, Efficient Implementation of a van der Waals Density Functional: Application to
Double-Wall Carbon Nanotubes, Phys. Rev. Lett., 2009, 103, 096102.

37 J. Klimeš, D. R. Bowler and A. Michaelides, van der Waals Density Functionals Applied to Solids, Phys. Rev. B: Condens. Matter Mater. Phys., 2011, 83, 195131.

38 J. Ontaneda, A. Singh, U. V. Waghmare and R. GrauCrespo, Origin of the Monolayer Raman Signature in Hexagonal Boron Nitride: A First Principles Analysis, J. Phys.: Condens. Matter, 2018, 30, 185701.

39 Z. Ni, Q. Liu, K. Tang, J. Zheng, J. Zhou, R. Qin, Z. Gao, D. $\mathrm{Yu}$ and J. Lu, Tunable Bandgap in Silicene and Germanene, Nano Lett., 2012, 12, 113-118. 